\title{
Parametric Cubic Spline Interpolating Surfaces for Arbitrary Polygonal Meshes
}

This paper was downloaded from TechRxiv (https://www.techrxiv.org).

\section{LICENSE}

CC BY-NC-SA 4.0

SUBMISSION DATE / POSTED DATE

20-01-2022 / 01-02-2022

\section{CITATION}

LIAN, JIAN-AO (2022): Parametric Cubic Spline Interpolating Surfaces for Arbitrary Polygonal Meshes. TechRxiv. Preprint. https://doi.org/10.36227/techrxiv.18820877.v1

$\mathrm{DOI}$ 


\title{
Parametric Cubic Spline Interpolating Surfaces for Arbitrary Polygonal Meshes
}

\author{
Jian-ao Lian, Senior Member, IEEE \\ Math. Dept. \\ Prairie View A\&M University \\ Prairie View, Texas, USA \\ unurbs@yahoo.com
}

\begin{abstract}
A fast algorithm for generating first order geometrically differentiable surfaces for arbitrary polygonal meshes by using parametric bicubic spline interpolation is established. It can be applied to any polygonal mesh as a quadrilateralization preprocessing can be performed if not all polygons of the given polygonal mesh are quadrilaterals. If needed, the resulting first order geometrically differentiable surface can create and provide point clouds with any desirable density based on available polygonal meshes. Various examples are demonstrated.

Index Terms-Computer graphics, interpolation, point cloud, spline and piecewise polynomial, surface fitting
\end{abstract}

\section{INTRODUCTION}

Three-dimensional (3d) scanners are widely used nowadays for scanning $3 \mathrm{~d}$ models, while computer-generated imagery (CGI) uses $3 \mathrm{~d}$ models for video games, animation films, and virtual reality modeling. In general, these $3 \mathrm{~d}$ models are stored in triangular meshes of various different file formats, such as 3DS, IGS, OBJ, PLY, STEP, STL, just name a few. The variety of formats are just specific to CAD proprietary software and for optimization purposes. However, for simplicity, PLY file format, with only its vertex and face lists included but its header omitted, will be used throughout this paper.

Though the notion spline was first introduced by I.J. Schoenberg in 1946 [12], both univariate and multivariate splines have been extensively and well developed since then, cf., e.g. [3], [4], [5], and [9]. Not only are they convenient mathematical tools and included in most of today's Numerical Analysis textbooks, but they are still being investigated and applied in many areas. Similar notions, such as bicubic splines, bivariate splines, tensor-product splines and spline wavelets, still constantly appear in modern scientific spline related studies and have found numerous successful engineering applications. Recently, an equidistant tool path planning strategy on curved freeform surfaces, using an inverse interpolation scheme of cubic spline parameterization, for robotic machining tasks, was proposed in [1]. An algorithm for numerical Hilbert transform of functions represented by cubic and exponential splines was developed in [6], and used for causal interpolation of characteristic impedance of microstrip line over conductive substrate. A 2D surface reconstruction from discretization points using cubic splines was studied in [7] for domain discretization in numerical simulations. A scheme of $B$-spline calculation for $G^{2}$ continuous trajectory generation was proposed in [10]. A simultaneous localization and mapping (SLAM) based technique that builds on top of a $B$-spline surface map was presented in [11]. A fast and robust method to estimate the ground surface from LiDAR (light detection and ranging) measurements on an automated vehicle, where the ground surface was modeled as a uniform $B$-spline, was proposed in [16] Neural splines for $3 \mathrm{~d}$ surface reconstruction that is based on random feature kernels arising from ReLU (rectified linear unit) networks was presented in [17]. An adaptive state estimation method by using cubic spline interpolation for particle filter tracking was presented in [18]. A rational bi-quartic spline interpolation scheme was constructed in $[8]$ and used for image enlargement. Local average interpolating splines for building surfaces were created in [13] to data from UAV's (unmanned aerial vehicle) aerial and satellite imagery. A new local spline quasi-interpolant was constructed for fitting $3 \mathrm{~d}$ data defined on the sphere-like surface in [2] and was successfully applied to medical imaging.

The main objective of this paper is to establish a geometrically first order differentiable surface for an arbitrary $3 \mathrm{~d}$ polygonal mesh (PM) by using PArametric biCubic Spline (PACS). High order bivariate splines could also be feasible but that leaves too many degrees of freedom to determine.

The outline of the paper is arranged as follows. To facilitate an easy presentation, PACS uniquely determined from 16 control points will be re-visited in Section II] with first order geometrically differentiable connections, called $G^{1}$, between two PACS on an edge and multiple PACS around a vertex being elucidated in Section III The main result for generating a PArametric biCubic Spline Interpolation Surface (PACSIS) for any PM will be laid out in Section IV To emphasize a PACSIS determined by 16 control points for each quad, a pseudo PLY format for any PACSIS will be introduced in Section $\mathrm{V}$ The fast algorithm will be demonstrated in Section VI, and Section VII incorporates the conclusions.

\section{PARAMETRIC BICUBIC SPLINES}

Unless otherwise specified, the current study is focused on bicubic splines. Denote by $b_{3, k}(t)$ the cubic Bézier bases or Bernstein polynomials, namely:

$$
b_{3, k}(t)=\left(\begin{array}{l}
3 \\
k
\end{array}\right) t^{k}(1-t)^{3-k}, k=0, \ldots, 3 .
$$




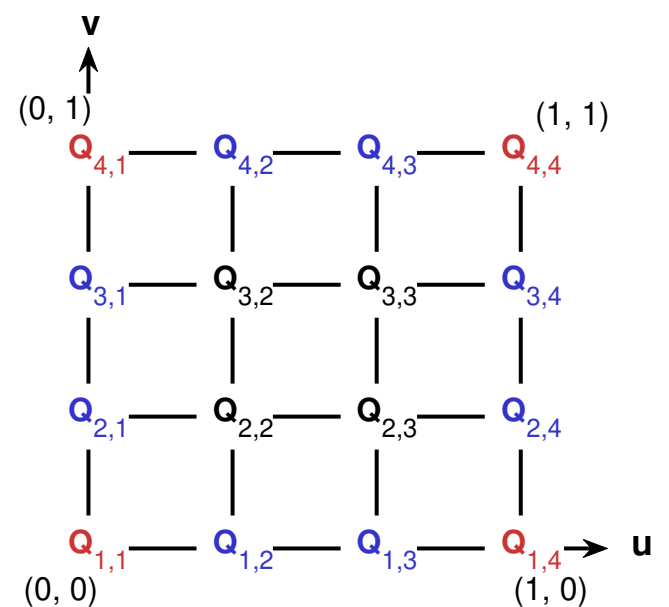

Fig. 1: Parametric domain and control points of a PACS with parameters $(u, v) \in[0,1]^{2}$.

With 16 control points $\mathbf{Q}_{j, k} \in \mathbb{R}^{3}, j, k=1, \ldots, 4$, arranged by the square matrix $\mathbf{Q}$ :

$$
\mathbf{Q}=\left[\begin{array}{llll}
\mathbf{Q}_{4,1} & \mathbf{Q}_{4,2} & \mathbf{Q}_{4,3} & \mathbf{Q}_{4,4} \\
\mathbf{Q}_{3,1} & \mathbf{Q}_{3,2} & \mathbf{Q}_{3,3} & \mathbf{Q}_{3,4} \\
\mathbf{Q}_{2,1} & \mathbf{Q}_{2,2} & \mathbf{Q}_{2,3} & \mathbf{Q}_{2,4} \\
\mathbf{Q}_{1,1} & \mathbf{Q}_{1,2} & \mathbf{Q}_{1,3} & \mathbf{Q}_{1,4}
\end{array}\right]
$$

a $2 \mathrm{~d}$ bicubic Bézier surface, denoted by $\mathbf{B}(u, v)$, has the parametric form of

$$
\begin{gathered}
\mathbf{B}(u, v)=\sum_{k=0}^{3} \sum_{j=0}^{3} \mathbf{Q}_{4-k, j+1} b_{3, j}(u) b_{3,3-k}(v) \\
=\left[b_{3,3}(v) b_{3,2}(v) b_{3,1}(v) b_{3,0}(v)\right] \mathbf{Q}\left[\begin{array}{l}
b_{3,0}(u) \\
b_{3,1}(u) \\
b_{3,2}(u) \\
b_{3,3}(u)
\end{array}\right],
\end{gathered}
$$

for $(u, v) \in[0,1]^{2}$. See Fig. 11 for an illustration of the parametric domain and 16 control points of a PACS. The $\mathbf{B}(u, v)$ in (3) will be called a PACS, which is completely determined by the 16 points (or $3 \mathrm{~d}$ vectors) in (2). It is rotation invariant, e.g., $\mathbf{B}(u, v)=\mathbf{B}(1-v, u)$. In addition, observe that $\mathbf{B}(u, v)$ interpolates tangent planes as well as values at the four corner control points, i.e.,

$$
\begin{array}{ll}
\mathbf{B}(0,0)=\mathbf{Q}_{1,1}, & \mathbf{B}(1,0)=\mathbf{Q}_{1,4} \\
\mathbf{B}(0,1)=\mathbf{Q}_{4,1}, & \mathbf{B}(1,1)=\mathbf{Q}_{4,4}
\end{array}
$$

and, with $\nabla=\left[\frac{\partial}{\partial u} \frac{\partial}{\partial v}\right]^{\top}$,

$$
\begin{aligned}
& \nabla \mathbf{B}(0,0)=3\left[\begin{array}{l}
\mathbf{Q}_{1,2}-\mathbf{Q}_{1,1} \\
\mathbf{Q}_{2,1}-\mathbf{Q}_{1,1}
\end{array}\right], \\
& \nabla \mathbf{B}(1,0)=3\left[\begin{array}{l}
\mathbf{Q}_{1,4}-\mathbf{Q}_{1,3} \\
\mathbf{Q}_{2,4}-\mathbf{Q}_{1,4}
\end{array}\right], \\
& \nabla \mathbf{B}(0,1)=3\left[\begin{array}{l}
\mathbf{Q}_{4,2}-\mathbf{Q}_{4,1} \\
\mathbf{Q}_{4,1}-\mathbf{Q}_{3,1}
\end{array}\right], \\
& \nabla \mathbf{B}(1,1)=3\left[\begin{array}{l}
\mathbf{Q}_{4,4}-\mathbf{Q}_{4,3} \\
\mathbf{Q}_{4,4}-\mathbf{Q}_{3,4}
\end{array}\right] .
\end{aligned}
$$

See Fig. 2 for an example of PACS.

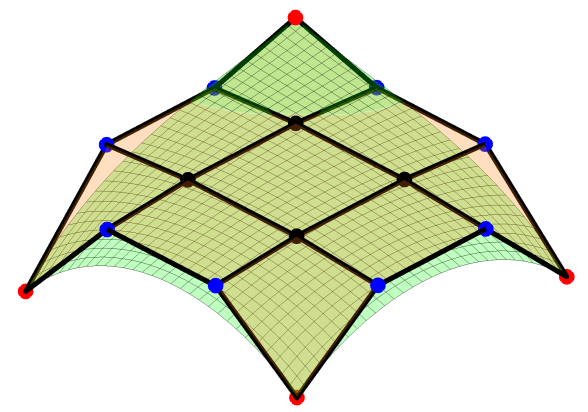

Fig. 2: A PACS with its 16 control points included.

\section{III. $G^{1}$ CONNECTION BETWEEN TWO ADJACENT PACS}

Let $\mathbf{B}_{1}(u, v)$ and $\mathbf{B}_{2}(u, v)$ be two adjacent PACS, i.e.,

$$
\begin{aligned}
& \mathbf{B}_{1}(u, v)=\sum_{k=0}^{3} \sum_{j=0}^{3} \mathbf{P}_{4-k, j+1} b_{3, j}(u) b_{3,3-k}(v), \\
& \mathbf{B}_{2}(u, v)=\sum_{k=0}^{3} \sum_{j=0}^{3} \mathbf{Q}_{4-k, j+1} b_{3, j}(u) b_{3,3-k}(v),
\end{aligned}
$$

for $(u, v) \in[0,1]^{2}$, with parameter domains shown in Fig. 3 All continuity conditions, when $\mathbf{B}_{1}(u, v)$ and $\mathbf{B}_{2}(u, v)$ are connected, can be expressed exactly by their control points along the shared edge. For parametric surfaces, the $G^{0}$ continuity is the common $C^{0}$ continuity. However, $G^{1}$ continuity means two surfaces share a common normal direction of their tangent planes at all points on their shared edge; and three or more PACS share a common vertex having the same normal direction of all tangent planes at the vertex.

Lemma 1 ( $G^{0}$ Continuity): Two pieces of PACS in Fig. 3 are $G^{0}$ connected if and only if

$$
\mathbf{Q}_{k, 1}=\mathbf{P}_{k, 4}, \quad k=1, \ldots, 4 \text {. }
$$




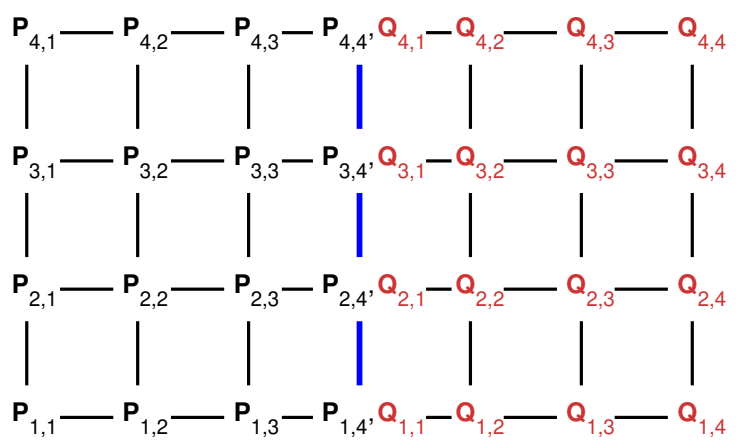

Fig. 3: Control points of two adjacent PACS.

Proof: It follows from $(6--7)$ that

$$
\begin{aligned}
& \mathbf{B}_{1}(1, v)=\sum_{k=0}^{3} \mathbf{P}_{4-k, 4} b_{3,3-k}(v), \\
& \mathbf{B}_{2}(0, v)=\sum_{k=0}^{3} \mathbf{Q}_{4-k, 1} b_{3,3-k}(v),
\end{aligned}
$$

so that $\mathbf{B}_{1}(u, v)$ and $\mathbf{B}_{2}(u, v)$ in (6)-(7) are $G^{0}$ connected if and only if $\mathbf{B}_{1}(1, v)=\mathbf{B}_{2}(0, v), v \in[0,1]$, i.e., 8 .

Moreover, the $G^{1}$ continuity can be determined from the following.

Lemma 2 ( $G^{1}$ Continuity): The $G^{1}$ continuity between $\mathbf{B}_{1}(u, v)$ and $\mathbf{B}_{2}(u, v)$ in (6)-77) along their common edge requires both $(8)$ and that the normal direction at any point on the common edge parallel, i.e.

$$
\left.\left(\frac{\partial \mathbf{B}_{1}}{\partial u} \times \frac{\partial \mathbf{B}_{1}}{\partial v}\right)\right|_{u=1} \|\left.\left(\frac{\partial \mathbf{B}_{2}}{\partial u} \times \frac{\partial \mathbf{B}_{2}}{\partial v}\right)\right|_{u=0} .
$$

In particular, the 3 vectors $\mathbf{P}_{1,4}-\mathbf{P}_{1,3}, \mathbf{Q}_{1,2}-\mathbf{P}_{1,4}$, and $\mathbf{P}_{2,4}-\mathbf{P}_{1,4}$ and the 3 vectors $\mathbf{P}_{4,4}-\mathbf{P}_{4,3}, \mathbf{P}_{4,4}-\mathbf{P}_{3,4}$, and $\mathbf{Q}_{4,2}-\mathbf{P}_{4,4}$ are coplanar.

Proof: It also follows from (6)-(7) that

$$
\begin{gathered}
\left.\frac{1}{3} \frac{\partial \mathbf{B}_{1}}{\partial u}\right|_{u=1} \\
=\left(\mathbf{P}_{4,4}-\mathbf{P}_{4,3}\right) b_{3,3}(v)+\left(\mathbf{P}_{3,4}-\mathbf{P}_{3,3}\right) b_{3,2}(v) \\
\quad+\left(\mathbf{P}_{2,4}-\mathbf{P}_{2,3}\right) b_{3,1}(v)+\left(\mathbf{P}_{1,4}-\mathbf{P}_{1,3}\right) b_{3,0}(v), \\
\left.\frac{1}{3} \frac{\partial \mathbf{B}_{1}}{\partial v}\right|_{u=1}=v^{2} \mathbf{P}_{4,4}+v(2-3 v) \mathbf{P}_{3,4} \\
\quad+(1-v)(1-3 v) \mathbf{P}_{2,4}-(1-v)^{2} \mathbf{P}_{1,4}, \\
\left.\frac{1}{3} \frac{\partial \mathbf{B}_{2}}{\partial u}\right|_{u=0} \quad\left(\mathbf{Q}_{4,2}-\mathbf{Q}_{4,1}\right) b_{3,3}(v)+\left(\mathbf{Q}_{3,2}-\mathbf{Q}_{3,1}\right) b_{3,2}(v) \\
\quad+\left(\mathbf{Q}_{2,2}-\mathbf{Q}_{2,1}\right) b_{3,1}(v)+\left(\mathbf{Q}_{1,2}-\mathbf{Q}_{1,1}\right) b_{3,0}(v), \\
\left.\frac{1}{3} \frac{\partial \mathbf{B}_{2}}{\partial v}\right|_{u=0}=v^{2} \mathbf{Q}_{4,1}+v(2-3 v) \mathbf{Q}_{3,1} \\
\quad+(1-v)(1-3 v) \mathbf{Q}_{2,1}-(1-v)^{2} \mathbf{Q}_{1,1} .
\end{gathered}
$$

Hence,

$$
\begin{aligned}
\mathbf{N}_{1}(v)=\left.\frac{1}{9}\left(\frac{\partial \mathbf{B}_{1}}{\partial u} \times \frac{\partial \mathbf{B}_{1}}{\partial v}\right)\right|_{u=1} \\
=\left[\mathbf{P}_{1,4}-\mathbf{P}_{1,3}+3 v\left(\mathbf{P}_{2,4}-\mathbf{P}_{2,3}-\mathbf{P}_{1,4}+\mathbf{P}_{1,3}\right)\right. \\
+3 v^{2}\left(\mathbf{P}_{1,4}-\mathbf{P}_{1,3}-2 \mathbf{P}_{2,4}+2 \mathbf{P}_{2,3}+\mathbf{P}_{3,4}-\mathbf{P}_{3,3}\right) \\
+v^{3}\left(-\mathbf{P}_{1,4}+\mathbf{P}_{1,3}+3 \mathbf{P}_{2,4}-3 \mathbf{P}_{2,3}\right. \\
\left.\left.\quad \quad-3 \mathbf{P}_{3,4}+3 \mathbf{P}_{3,3}+\mathbf{P}_{4,4}-\mathbf{P}_{4,3}\right)\right] \\
\quad \times\left[\mathbf{P}_{2,4}-\mathbf{P}_{1,4}+2 v\left(\mathbf{P}_{1,4}-2 \mathbf{P}_{2,4}+\mathbf{P}_{3,4}\right)\right. \\
\left.\quad+v^{2}\left(-\mathbf{P}_{1,4}+3 \mathbf{P}_{2,4}-3 \mathbf{P}_{3,4}+\mathbf{P}_{4,4}\right)\right]
\end{aligned}
$$

and

$$
\begin{aligned}
\mathbf{N}_{2}(v) & =\left.\frac{1}{9}\left(\frac{\partial \mathbf{B}_{2}}{\partial u} \times \frac{\partial \mathbf{B}_{2}}{\partial v}\right)\right|_{u=0} \\
= & {\left[\mathbf{Q}_{1,2}-\mathbf{Q}_{1,1}+3 v\left(\mathbf{Q}_{2,2}-\mathbf{Q}_{2,1}-\mathbf{Q}_{1,2}+\mathbf{Q}_{1,1}\right)\right.} \\
& +3 v^{2}\left(\mathbf{Q}_{1,2}-\mathbf{Q}_{1,1}-2 \mathbf{Q}_{2,2}+2 \mathbf{Q}_{2,1}+\mathbf{Q}_{3,2}-\mathbf{Q}_{3,1}\right) \\
& +v^{3}\left(-\mathbf{Q}_{1,2}+\mathbf{Q}_{1,1}+3 \mathbf{Q}_{2,2}-3 \mathbf{Q}_{2,1}\right. \\
& \left.\left.\quad-3 \mathbf{Q}_{3,2}+3 \mathbf{Q}_{3,1}+\mathbf{Q}_{4,2}-\mathbf{Q}_{4,1}\right)\right] \\
\times & {\left[\mathbf{Q}_{2,1}-\mathbf{Q}_{1,1}+2 v\left(\mathbf{Q}_{1,1}-2 \mathbf{Q}_{2,1}+\mathbf{Q}_{3,1}\right)\right.} \\
& \left.+v^{2}\left(-\mathbf{Q}_{1,1}+3 \mathbf{Q}_{2,1}-3 \mathbf{Q}_{3,1}+\mathbf{Q}_{4,1}\right)\right] .
\end{aligned}
$$

Expand (10) and (11) and rewrite them as

$$
\begin{aligned}
& \mathbf{N}_{1}(v)=\sum_{k=0}^{5} \mathbf{n}_{1, k} v^{k}, \\
& \mathbf{N}_{2}(v)=\sum_{k=0}^{5} \mathbf{n}_{2, k} v^{k} .
\end{aligned}
$$

Hence, the $G^{1}$ continuity between $\mathbf{B}_{1}(u, v)$ and $\mathbf{B}_{2}(u, v)$ means both 99 and, with $\mathbf{N}_{1}(v)$ and $\mathbf{N}_{2}(v)$ in $10 p-\sqrt{13}$,

$$
\mathbf{N}_{1}(v) \| \mathbf{N}_{2}(v), \quad v \in[0,1] .
$$

In particular, when $v=0$,

$$
\mathbf{n}_{1,0} \| \mathbf{n}_{2,0},
$$

which is, due to 8 ,

$$
\begin{aligned}
& \mathbf{n}_{1,0}=\left(\mathbf{P}_{1,4}-\mathbf{P}_{1,3}\right) \times\left(\mathbf{P}_{2,4}-\mathbf{P}_{1,4}\right), \\
& \mathbf{n}_{2,0}=\left(\mathbf{Q}_{1,2}-\mathbf{P}_{1,4}\right) \times\left(\mathbf{P}_{2,4}-\mathbf{P}_{1,4}\right),
\end{aligned}
$$

so that $\mathbf{N}_{1}(1) \| \mathbf{N}_{2}(1)$ is equivalent to the 3 vectors $\mathbf{P}_{1,4}-$ $\mathbf{P}_{1,3}, \mathbf{Q}_{1,2}-\mathbf{P}_{1,4}$, and $\mathbf{P}_{2,4}-\mathbf{P}_{1,4}$ are coplanar. Similarly, when $v=1$, the requirement $\mathbf{N}_{1}(1) \| \mathbf{N}_{2}(1)$ is equivalent to the 3 vectors $\mathbf{P}_{4,4}-\mathbf{P}_{4,3}, \mathbf{P}_{4,4}-\mathbf{P}_{3,4}$, and $\mathbf{Q}_{4,2}-\mathbf{P}_{4,4}$ are coplanar.

\section{Main Results}

As two introductory examples, the first PLY data file

41

$\begin{array}{lll}1 & 0 & 0\end{array}$

$\begin{array}{lll}1 & 1 & 0\end{array}$

$0 \begin{array}{lll}0 & 1 & 0\end{array}$ 
$\begin{array}{lll}0 & 0 & 1\end{array}$

$\begin{array}{lllll}4 & 0 & 1 & 2 & 3\end{array}$

has 4 vertices and 1 quadrilateral, while the second PLY file

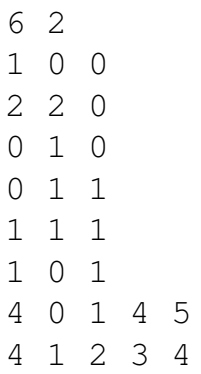

has 6 vertices and 2 quadrilaterals. The question is: How to construct $G^{1}$ PACSIS that interpolate the 4 vertices and 6 vertices in the two PLY files? The solutions are not unique. Fig. 4 displays a PACSIS for the 1-quad PLY polygon; and Fig. 5 shows a $G^{1}$ PACSIS for the 2-quad PLY PM.
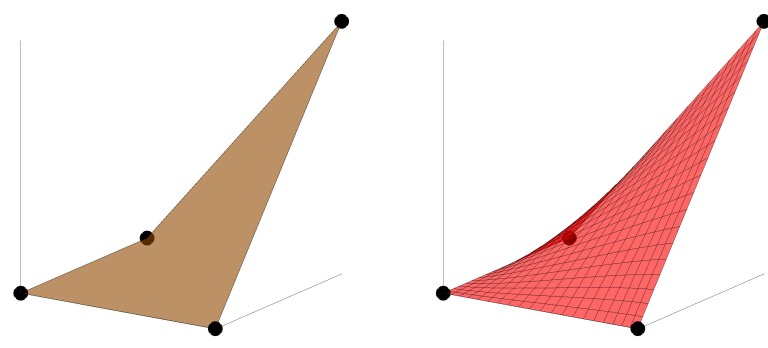

Fig. 4: One quad mesh and its resulting one piece PACSIS.
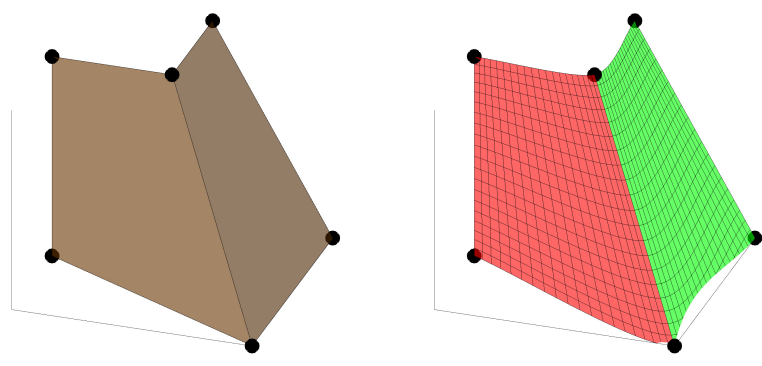

Fig. 5: Two quad mesh and its resulting two piece PACSIS.

First, the following result shows sufficient conditions to get a $G^{1}$ PACSIS for two adjacent PACS. To adapt to more general settings, notations for control points are slightly changed.

Theorem 1 ( $G^{1}$ PACSIS from two adjacent PACS): Let $\mathbf{B}_{1}(u, v)$ and $\mathbf{B}_{2}(u, v)$ be the two adjacent PACS in Fig. 6 that interpolate the 6 points $\mathbf{P}_{i, j}, i=1,2$, and $j=1,2,3$. Let $\left\{\mathbf{d}_{1,1}, \mathbf{d}_{1,2}\right\}$ and $\left\{\mathbf{d}_{2,1}, \mathbf{d}_{2,2}\right\}$ represent the two directions on the tangent planes at $\mathbf{P}_{1,2}$ and $\mathbf{P}_{2,2}$, respectively, e.g.,

$$
\begin{aligned}
\mathbf{d}_{1,1} & =\mathbf{P}_{1,3}-\mathbf{P}_{1,1}, \\
\mathbf{d}_{1,2} & =\mathbf{P}_{2,2}-\mathbf{P}_{1,2}, \\
\mathbf{d}_{2,1} & =\mathbf{P}_{2,3}-\mathbf{P}_{2,1}, \\
\mathbf{d}_{2,2} & =\mathbf{P}_{1,2}-\mathbf{P}_{2,2} .
\end{aligned}
$$

Set

$$
\begin{aligned}
& \mathbf{Q}_{1,1,3}=\mathbf{P}_{1,2}-\alpha_{1} \mathbf{d}_{1,1}, \\
& \mathbf{Q}_{2,1,2}=\mathbf{P}_{1,2}+\alpha_{1} \mathbf{d}_{1,1}, \\
& \mathbf{Q}_{1,2,4}=\mathbf{Q}_{2,2,1}=\mathbf{P}_{1,2}+\alpha_{1} \mathbf{d}_{1,2}, \\
& \mathbf{Q}_{1,2,3}=\mathbf{P}_{1,2}-\alpha_{1} \mathbf{d}_{1,1}+\alpha_{1} \mathbf{d}_{1,2}, \\
& \mathbf{Q}_{2,2,2}=\mathbf{P}_{1,2}+\alpha_{1} \mathbf{d}_{1,1}+\alpha_{1} \mathbf{d}_{1,2}, \\
& \mathbf{Q}_{1,4,3}=\mathbf{P}_{2,2}-\alpha_{2} \mathbf{d}_{2,1}, \\
& \mathbf{Q}_{2,4,2}=\mathbf{P}_{2,2}+\alpha_{2} \mathbf{d}_{2,1}, \\
& \mathbf{Q}_{1,3,4}=\mathbf{Q}_{2,3,1}=\mathbf{P}_{2,2}+\alpha_{2} \mathbf{d}_{2,2}, \\
& \mathbf{Q}_{1,3,3}=\mathbf{P}_{2,2}-\alpha_{2} \mathbf{d}_{2,1}+\alpha_{2} \mathbf{d}_{2,2}, \\
& \mathbf{Q}_{2,3,2}=\mathbf{P}_{2,2}+\alpha_{2} \mathbf{d}_{2,1}+\alpha_{2} \mathbf{d}_{2,2},
\end{aligned}
$$

where $\alpha_{1}$ and $\alpha_{2}$ are nonzero constants. Then $\mathbf{B}_{1}(u, v)$ and $\mathbf{B}_{2}(u, v)$ are $G^{1}$ connected.

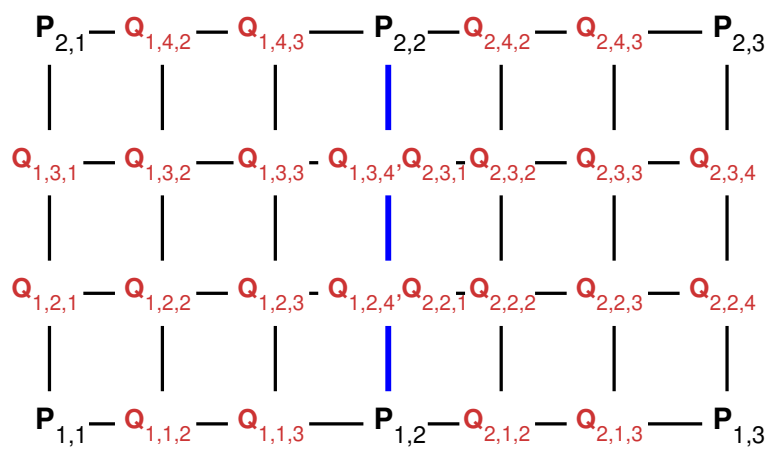

Fig. 6: A PACSIS with two adjacent PACS.

Proof: It follows by substituting (18)-(31) into

$$
\left.\frac{1}{3} \frac{\partial \mathbf{B}_{1}}{\partial u}\right|_{u=1},\left.\frac{1}{3} \frac{\partial \mathbf{B}_{2}}{\partial u}\right|_{u=0},\left.\frac{1}{3} \frac{\partial \mathbf{B}_{1}}{\partial v}\right|_{u=1},\left.\frac{1}{3} \frac{\partial \mathbf{B}_{2}}{\partial v}\right|_{u=0},
$$

that

$$
\begin{aligned}
& \left.\frac{1}{3} \frac{\partial \mathbf{B}_{1}}{\partial u}\right|_{u=1} \\
& =\mathbf{P}_{1,2}-\mathbf{Q}_{1,1,3}+3 v\left(\mathbf{Q}_{1,2,4}-\mathbf{Q}_{1,2,3}-\mathbf{P}_{1,2}+\mathbf{Q}_{1,1,3}\right) \\
& \quad+3 v^{2}\left(\mathbf{P}_{1,2}-\mathbf{Q}_{1,1,3}-2 \mathbf{Q}_{1,2,4}\right. \\
& \left.\quad+2 \mathbf{Q}_{1,2,3}+\mathbf{Q}_{1,3,4}-\mathbf{Q}_{1,3,3}\right) \\
& \quad+v^{3}\left(-\mathbf{P}_{1,2}+\mathbf{Q}_{1,1,3}+3 \mathbf{Q}_{1,2,4}-3 \mathbf{Q}_{1,2,3}\right. \\
& \left.\quad-3 \mathbf{Q}_{1,3,4}+3 \mathbf{Q}_{1,3,3}+\mathbf{P}_{2,2}-\mathbf{Q}_{1,4,3}\right) \\
& =\alpha_{1} \mathbf{d}_{1,1}+3 v^{2}\left(-\alpha_{1} \mathbf{d}_{1,1}+\alpha_{2} \mathbf{d}_{2,1}\right) \\
& \quad+2 v^{3}\left(\alpha_{1} \mathbf{d}_{1,1}-\alpha_{2} \mathbf{d}_{2,1}\right)
\end{aligned}
$$




$$
\begin{aligned}
\left.\frac{1}{3} \frac{\partial \mathbf{B}_{2}}{\partial u}\right|_{u=0} & \\
= & \mathbf{Q}_{2,1,2}-\mathbf{P}_{1,2}+3 v\left(\mathbf{Q}_{2,2,2}-\mathbf{Q}_{2,2,1}-\mathbf{Q}_{2,1,2}+\mathbf{P}_{1,2}\right) \\
& +3 v^{2}\left(\mathbf{Q}_{2,1,2}-\mathbf{P}_{1,2}-2 \mathbf{Q}_{2,2,2}\right. \\
& \left.+2 \mathbf{Q}_{2,2,1}+\mathbf{Q}_{2,3,2}-\mathbf{Q}_{2,3,1}\right) \\
+ & v^{3}\left(-\mathbf{Q}_{2,1,2}+\mathbf{P}_{1,2}+3 \mathbf{Q}_{2,2,2}-3 \mathbf{Q}_{2,2,1}\right. \\
& \left.-3 \mathbf{Q}_{2,3,2}+3 \mathbf{Q}_{2,3,1}+\mathbf{Q}_{2,4,2}-\mathbf{P}_{2,2}\right) \\
= & \alpha_{1} \mathbf{d}_{1,1}+3 v^{2}\left(-\alpha_{1} \mathbf{d}_{1,1}+\alpha_{2} \mathbf{d}_{2,1}\right) \\
& +2 v^{3}\left(\alpha_{1} \mathbf{d}_{1,1}-\alpha_{2} \mathbf{d}_{2,1}\right)
\end{aligned}
$$

$$
\begin{aligned}
\left.\frac{1}{3} \frac{\partial \mathbf{B}_{1}}{\partial v}\right|_{u=1} \\
=\mathbf{Q}_{1,2,4}-\mathbf{P}_{1,2}+2 v\left(\mathbf{P}_{1,2}-2 \mathbf{Q}_{1,2,4}+\mathbf{Q}_{1,3,4}\right) \\
\quad+v^{2}\left(-\mathbf{P}_{1,2}+3 \mathbf{Q}_{1,2,4}-3 \mathbf{Q}_{1,3,4}+\mathbf{P}_{2,2}\right) \\
=\alpha_{1} \mathbf{d}_{1,2}+2 v\left(-\mathbf{P}_{1,2}+\mathbf{P}_{2,2}-2 \alpha_{1} \mathbf{d}_{1,2}+\alpha_{2} \mathbf{d}_{2,2}\right) \\
\quad+v^{2}\left(2 \mathbf{P}_{1,2}-2 \mathbf{P}_{2,2}+3 \alpha_{1} \mathbf{d}_{1,2}-3 \alpha_{2} \mathbf{d}_{2,2}\right)
\end{aligned}
$$

$$
\begin{aligned}
\left.\frac{1}{3} \frac{\partial \mathbf{B}_{2}}{\partial v}\right|_{u=0} \\
=\mathbf{Q}_{2,2,1}-\mathbf{P}_{1,2}+2 v\left(\mathbf{P}_{1,2}-2 \mathbf{Q}_{2,2,1}+\mathbf{Q}_{2,3,1}\right) \\
\quad+v^{2}\left(-\mathbf{P}_{1,2}+3 \mathbf{Q}_{2,2,1}-3 \mathbf{Q}_{2,3,1}+\mathbf{P}_{2,2}\right) \\
=\alpha_{1} \mathbf{d}_{1,2}+2 v\left(-\mathbf{P}_{1,2}+\mathbf{P}_{2,2}-2 \alpha_{1} \mathbf{d}_{1,2}+\alpha_{2} \mathbf{d}_{2,2}\right) \\
\quad+v^{2}\left(2 \mathbf{P}_{1,2}-2 \mathbf{P}_{2,2}+3 \alpha_{1} \mathbf{d}_{1,2}-3 \alpha_{2} \mathbf{d}_{2,2}\right) .
\end{aligned}
$$

Hence, (32)-35 leads to

$$
\left.\frac{\partial \mathbf{B}_{1}}{\partial u}\right|_{u=1}=\left.\frac{\partial \mathbf{B}_{2}}{\partial u}\right|_{u=0},\left.\quad \frac{\partial \mathbf{B}_{1}}{\partial v}\right|_{u=1}=\left.\frac{\partial \mathbf{B}_{2}}{\partial v}\right|_{u=0},
$$

which implies (14), or the requirement for the tangent plane at any point along the shared edge match.

Observe that the remaining control points in Fig. 6 are not given in Theorem 1, as they could be arbitrary or could not affect the $G^{1}$ connection of the two PACSIS. Their values in general will be determined if there are more quadrilaterals for a generic PM. This will be reflected in the following.

Theorem $2\left(G^{1}\right.$ PACSIS from any PM): For any PM with quadrilaterals, let $\mathbf{P}_{i}$ be a typical vertex; let $\mathbf{n}_{i}$ be the normal direction of the tangent plane at the $i^{\text {th }}$ vertex $\mathbf{P}_{i}$; let $\mathbf{B}_{i, j}(u, v)$ be a PACS that includes $\mathbf{P}_{i}$ as one of its four vertices; and let $\mathbf{P}_{i, j, 1}$ and $\mathbf{P}_{i, j, 2}$ be the other two vertices on the edges from the vertex $\mathbf{P}_{i}$, as shown in Fig. 7. Settle the three control points on $\mathbf{B}_{i, j}(u, v)$ around the vertex $\mathbf{P}_{i}$ by

$$
\begin{aligned}
& \mathbf{Q}_{i, j, 1}=\mathbf{P}_{i}+\alpha_{i} \mathbf{d}_{i, j, 1}, \\
& \mathbf{Q}_{i, j, 2}=\mathbf{P}_{i}+\alpha_{i} \mathbf{d}_{i, j, 2}, \\
& \mathbf{Q}_{i, j, 3}=\mathbf{P}_{i}+\alpha_{i} \mathbf{d}_{i, j, 1}+\alpha_{i} \mathbf{d}_{i, j, 2},
\end{aligned}
$$

where $\alpha_{i}$ is an appropriate constant called an elastic parameter, $\mathbf{d}_{i, j, 1}$ and $\mathbf{d}_{i, j, 2}$ are two vectors on the tangent plane at $\mathbf{P}_{i}$, with the normal direction $\mathbf{n}_{i}$, and parallel to $\mathbf{P}_{i, j, 1}-\mathbf{P}_{i}$ and $\mathbf{P}_{i, j, 2}-\mathbf{P}_{i}$. Then the resulting PACSIS is $G^{1}$ connected.
Proof: With a fixed elastic parameter and a fixed tangent plane around a vertex $\mathbf{P}_{i}$, it follows from Theorem 1 that any two adjacent PACS are connected in a $G^{1}$ fashion. So all PACS around the vertex $\mathbf{P}_{i}$ are $G^{1}$ connected; and all PACS of the resulting PACSIS is $G^{1}$ connected.

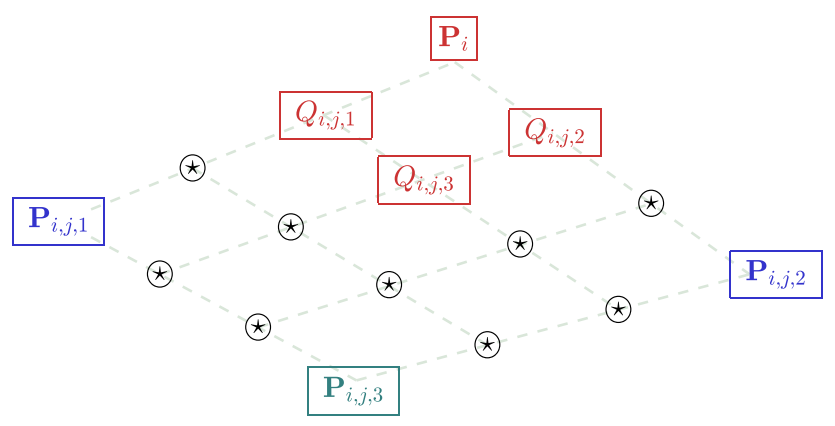

Fig. 7: PACSIS with new control points created at each vertex of a PM.

If not all polygons of a given PM are quadrilaterals, a quadrilateralization preprocessing is necessary. This can be done by adding a new control vertex of the polygon, which is in general the centroid of the polygon, and connecting each midpoint of an edge on the polygon to the new vertex.

Here is a detailed algorithm that describes how to settle all control points of a PM by using Theorem 2 .

\section{Algorithm.1 $G^{1}$ PACSIS for an arbitrary PM}

1) If not all polygons in the PM are quadrilaterals, perform the quadrilateralization preprocessing. Let $\mathbf{F}_{j}$ be the $j^{\text {th }}$ polygon (or face) of the PM, $n\left(\mathbf{F}_{j}\right)$ be the number of sides of the polygon $\mathbf{F}_{j}$, and $\mathbf{P}_{j, k}, k=1, \ldots, n\left(\mathbf{F}_{j}\right)$, be its vertices. Let $\mathbf{N}_{i}$ be the normal direction of the tangent plane at the vertex $\mathbf{P}_{i}$.

a) Evaluate the centroid of $\mathbf{F}_{j}$ :

$$
\mathbf{C}_{j}=\frac{1}{n\left(\mathbf{F}_{j}\right)} \sum_{k=1}^{n\left(\mathbf{F}_{j}\right)} \mathbf{P}_{j, k} .
$$

b) Let $\mathbf{M}_{j, \ell}$ be the midpoint of the edge from $\mathbf{P}_{j, \ell}$ to $\mathbf{P}_{j, \ell+1}$ :

$$
\mathbf{M}_{j, \ell}=\frac{1}{2}\left(\mathbf{P}_{j, \ell}+\mathbf{P}_{j, \ell+1}\right),
$$

for $\ell=1, \ldots, n\left(\mathbf{F}_{j}\right)$, where $\mathbf{P}_{j, n\left(\mathbf{F}_{j}\right)+1}=\mathbf{P}_{j, 1}$.

c) Create a new PM with only quadrilaterals from the given PM by (1) keeping all old vertices; (2) adding new vertices $\mathbf{C}_{j}, \mathbf{M}_{j, \ell}, \ell=1, \ldots, n\left(\mathbf{F}_{j}\right)$; and (3) abandoning all old faces but adding new faces

$$
\left\{\mathbf{C}_{j}, \mathbf{M}_{j, \ell}, \mathbf{P}_{j, \ell+1}, \mathbf{M}_{j, \ell+1}\right\},
$$

for $\ell=1, \ldots, n\left(\mathbf{F}_{j}\right)$, where $\mathbf{P}_{j, n\left(\mathbf{F}_{j}\right)+1}=\mathbf{P}_{j, 1}$ and $\mathbf{M}_{j, n\left(\mathbf{F}_{j}\right)+1}=\mathbf{M}_{j, 1}$.

By the way, if the original PM contains quadrilaterals only, this step is optional. 
2) Let $n_{V}$ be the total number of vertices of the (original or new) PM. Let $\mathbf{P}_{i}$ be the $i^{\text {th }}$ vertex of the (original or new) PM with quadrilaterals only, and $\mathbf{B}_{i, j}(u, v), j=$ $1, \ldots, n_{i}$, be the $n_{i}$ quads that share $\mathbf{P}_{i}$ as their common vertex. Let $\mathbf{T}_{i}$ be the tangent plane shared by all $n_{i}$ quads around the vertex (or initial interpolatory control point) $\mathbf{P}_{i}$. Determine the two directions of $\mathbf{T}_{i}$ by the following.

a) If $n_{i}=1$, let $\mathbf{P}_{i, 1}$ and $\mathbf{P}_{i, 2}$ be the 2 vertices of $\mathbf{B}_{i, 1}(u, v)$, with $\left(\mathbf{P}_{i}, \mathbf{P}_{i, 1}\right)$ and $\left(\mathbf{P}_{i}, \mathbf{P}_{i, 2}\right)$ being the two edges on $\mathbf{B}_{i, 1}(u, v)$. Then

$$
\begin{aligned}
\mathbf{d}_{i, 1} & =\mathbf{P}_{i, 1}-\mathbf{P}_{i}, \\
\mathbf{d}_{i, 2} & =\mathbf{P}_{i, 2}-\mathbf{P}_{i} .
\end{aligned}
$$

b) If $n_{i}=2$, let $\mathbf{P}_{i, 1,1}$ be the vertex on $\mathbf{B}_{i, 1}(u, v)$ and $\mathbf{P}_{i, 2,1}$ be the vertex on $\mathbf{B}_{i, 2}(u, v)$ so that $\left(\mathbf{P}_{i}, \mathbf{P}_{i, 1,1}\right)$ is an edge on $\mathbf{B}_{i, 1}(u, v)$ and $\left(\mathbf{P}_{i}, \mathbf{P}_{i, 2,1}\right)$ is an edge on $\mathbf{B}_{i, 2}(u, v)$. Let $\mathbf{P}_{i, 3}$ be vertex for both $\mathbf{B}_{i, 1}(u, v)$ and $\mathbf{B}_{i, 2}(u, v)$ so that $\left(\mathbf{P}_{i}, \mathbf{P}_{i, 3}\right)$ is their shared edge. Then

$$
\begin{aligned}
\mathbf{d}_{i, 1} & =\frac{1}{2}\left(\mathbf{P}_{i, 2,1}-\mathbf{P}_{i, 1,1}\right), \\
\mathbf{d}_{i, 2} & =\mathbf{P}_{i, 3}-\mathbf{P}_{i} .
\end{aligned}
$$

c) If $n_{i} \geq 3$, let $\mathbf{P}_{i, j, 1}$ and $\mathbf{P}_{i, j, 2}$ be the two vertices connecting to $\mathbf{P}_{i}$ for $\mathbf{B}_{i, j}(u, v), j=1, \ldots, n_{i}$. Find $\mathbf{N}_{i}$ through one of the following three scenarios. (1) By using the midpoints of all the diagonals $\left(\mathbf{P}_{i, j, 1}, \mathbf{P}_{i, j, 2}\right)$, form a matrix $A_{i, 1}$, constituting all of these midpoints as columns of $A_{i, 1}$. If $A_{i, 1} A_{i, 1}^{\top}$ is nonsingular, set

$$
\mathbf{N}_{i}=\left(A_{i, 1} A_{i, 1}^{\top}\right)^{-1} A_{i, 1} \mathbf{1}_{n_{i}},
$$

where $\mathbf{1}_{n_{i}}$ is the $n_{i}$-dimensional vector with all components as 1's. (2) If $A_{i, 1} A_{i, 1}^{\top}$ is singular, form a matrix $A_{i, 2}$, constituting all vertices of $\mathbf{P}_{i, j, 1}, \mathbf{P}_{i, j, 2}$, $j=1, \ldots, n_{i}$. If $A_{i, 2} A_{i, 2}^{\top}$ is nonsingular, set

$$
\mathbf{N}_{i}=\left(A_{i, 2} A_{i, 2}^{\top}\right)^{-1} A_{i, 2} \mathbf{1}_{2 n_{i}} .
$$

(3) If $A_{i, 2} A_{i, 2}^{\top}$ is singular, evaluate all vectors from the midpoint of $\left(\mathbf{P}_{i, 1,1}, \mathbf{P}_{i, 1,2}\right)$ to the midpoints of $\left(\mathbf{P}_{i, j, 1}, \mathbf{P}_{i, j, 2}\right), j=2, \ldots, n_{i}-1$. Select two nonparallel vectors, to be named $\mathbf{V}_{i, 1}$ and $\mathbf{V}_{i, 2}$, among the $n_{i}$ vectors. Finally, determine $\mathbf{N}_{i}$ by

$$
\mathbf{N}_{i}=\mathbf{V}_{i, 1} \times \mathbf{V}_{i, 2} .
$$

After $\mathbf{N}_{i}$ is evaluated, set

$$
\begin{aligned}
\mathbf{d}_{i, 1}= & \mathbf{W}_{i, j, 1}-\mathbf{P}_{i} \\
& -\left[\left(\mathbf{W}_{i, j, 1}-\mathbf{P}_{i}\right) \cdot \frac{\mathbf{N}_{i}}{\left\|\mathbf{N}_{i}\right\|}\right] \frac{\mathbf{N}_{i}}{\left\|\mathbf{N}_{i}\right\|}, \\
\mathbf{d}_{i, 2} & =\mathbf{W}_{i, j, 3}-\mathbf{P}_{i} \\
& -\left[\left(\mathbf{W}_{i, j, 3}-\mathbf{P}_{i}\right) \cdot \frac{\mathbf{N}_{i}}{\left\|\mathbf{N}_{i}\right\|}\right] \frac{\mathbf{N}_{i}}{\left\|\mathbf{N}_{i}\right\|} .
\end{aligned}
$$

3) As indicated in Theorem 2, for a typical quad $\mathbf{B}_{i, j}(u, v)$ that includes $\mathbf{P}_{i}$ as one of its four vertices, evaluate the three control points on $\mathbf{B}_{i, j}(u, v)$ that are close to the vertex $\mathbf{P}_{i}$ through (36)-38) for $i=1, \ldots, n_{V}$.

\section{PACSIS FILE FORMAT}

Observe that, with each quad's four vertices, Step 2 and Step 3 of Algorithm 1 are mainly for evaluating each quad's 12 additional control points. After all control points for all quads are calculated, the PACSIS is completely created. These control points are stored in a pseudo PLY file, which has the following format (with all header info omitted):

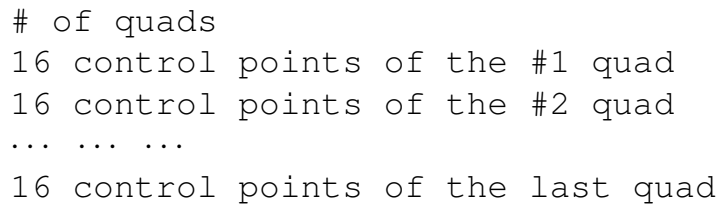

The corresponding PACSIS can be plotted from plotting all quads as rectangular patches in such a file. Due to the fact that each quad is a PACS, the PACSIS can be plotted as either a $G^{1}$ surface or a point cloud with as many points as one prefers.

As an example, the pseudo PLY file

16

$1.000 \quad 0.000 \quad 0.000 \quad 1.0390 .132-0.118$

$1.0670 .817-0.088 \quad 1.000 \quad 1.000 \quad 0.000$

$\begin{array}{llllll}0.763 & -0.039 & -0.039 & 0.803 & 0.092 & -0.158\end{array}$

$\begin{array}{llllllll}0.883 & 0.883 & -0.176 & 0.817 & 1.067 & -0.088\end{array}$

$\begin{array}{llllllll}0.250 & 0.000 & 0.000 & 0.250 & 0.250 & 0.000\end{array}$

$\begin{array}{lllllll}0.092 & 0.803 & -0.158 & 0.132 & 1.039 & -0.118\end{array}$

$\begin{array}{lllllllll}0.000 & 0.000 & 0.000 & 0.000 & 0.250 & 0.000\end{array}$

$\begin{array}{lllll}-0.039 & 0.763-0.039 & 0.000 & 1.000 & 0.000\end{array}$

(\& 720 numbers for remaining 15 quads)

represent a $G^{1}$ PACSIS of 16 quads, plotted in Fig. 8

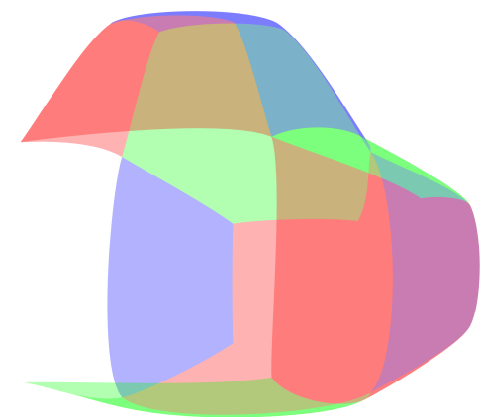

Fig. 8: A pseudo PLY file for a 16 piece $G^{1}$ PACSIS.

\section{IMPLEMENTATION}

Examples will be given in this section to demonstrate the fast performance of the PACSIS construction for any PM. 
Example 1. For the PLY file with 25 vertices and 16 quads,

2516

000

$\begin{array}{lll}1 & 0 & 0\end{array}$

$\begin{array}{lll}2 & 0 & 0 \\ 3 & 0 & 0\end{array}$

$\begin{array}{lll}3 & 0 & 0\end{array}$

400

$\begin{array}{lll}4 & 1 & 0\end{array}$

$\begin{array}{lll}4 & 2 & 0\end{array}$

430

$\begin{array}{lll}4 & 4 & 0\end{array}$

$\begin{array}{lll}3 & 4 & 0\end{array}$

240

$\begin{array}{lll}1 & 4 & 0\end{array}$

$\begin{array}{lll}0 & 4 & 0\end{array}$

$\begin{array}{lll}0 & 3 & 0\end{array}$

$\begin{array}{lll}0 & 2 & 0\end{array}$

$\begin{array}{lll}0 & 1 & 0\end{array}$

$\begin{array}{lll}1 & 1 & 0\end{array}$

$\begin{array}{lll}2 & 1 & 0\end{array}$

$\begin{array}{lll}3 & 1 & 0\end{array}$

$\begin{array}{lll}3 & 2 & 0\end{array}$

$\begin{array}{lll}3 & 3 & 0\end{array}$

230

$\begin{array}{lll}1 & 3 & 0\end{array}$

$\begin{array}{lll}1 & 2 & 0\end{array}$

$\begin{array}{lll}2 & 2 & 1\end{array}$

$\begin{array}{lllll}4 & 0 & 1 & 16 & 15\end{array}$

$\begin{array}{lllll}4 & 1 & 2 & 17 & 16\end{array}$

$\begin{array}{lllll}4 & 2 & 3 & 18 & 17\end{array}$

$\begin{array}{lllll}4 & 3 & 4 & 5 & 18\end{array}$

$\begin{array}{lllll}4 & 15 & 16 & 23 & 14\end{array}$

$\begin{array}{lllll}4 & 16 & 17 & 24 & 23\end{array}$

$\begin{array}{lllll}4 & 17 & 18 & 19 & 24\end{array}$

$\begin{array}{lllll}4 & 18 & 5 & 6 & 19\end{array}$

$\begin{array}{lllll}4 & 14 & 23 & 22 & 1\end{array}$

$\begin{array}{llllll}3 & 4 & 23 & 24 & 21 & 22\end{array}$

$\begin{array}{lllll}4 & 24 & 19 & 20 & 21\end{array}$

$\begin{array}{lllll}4 & 19 & 6 & 7 & 20\end{array}$

$\begin{array}{lllll}4 & 13 & 22 & 11 & 12\end{array}$

$\begin{array}{lllll}4 & 22 & 21 & 10 & 11\end{array}$

$\begin{array}{lllll}4 & 21 & 20 & 9 & 10\end{array}$

$4 \begin{array}{lllll}4 & 20 & 7 & 8 & 9\end{array}$

Fig. 9 shows the original PM and the resulting PACSIS.

Example 2. For the PLY file with 13 vertices and 3 hexagons,
$.501 .50 \quad .25$

$.50 \quad 1.50 \quad .50$

.50 .251 .50

$.25 \quad .50 \quad 1.50$

.50 .501 .50

$\begin{array}{lllllll}6 & 1 & 5 & 6 & 4 & 0 & 3\end{array}$

$\begin{array}{llllllll}6 & 7 & 2 & 3 & 1 & 8 & 9\end{array}$

$\begin{array}{lllllll}6 & 2 & 11 & 12 & 10 & 0 & 3\end{array}$

Fig. 10 shows the original PM with 13 vertices and 3 hexagons, its quadrilateralization, and the resulting $G^{1}$ PACSIS.

Example 3. For the PLY file of a closed seat shaped PM with 20 vertices and 18 quads,

2018

$\begin{array}{lll}0 & 0 & 0\end{array}$

$\begin{array}{lll}1 & 0 & 0\end{array}$

$\begin{array}{lll}2 & 0 & 0\end{array}$

$\begin{array}{lll}2 & 1 & 0\end{array}$

$\begin{array}{lll}1 & 1 & 0\end{array}$

$\begin{array}{lll}1 & 2 & 0\end{array}$

$\begin{array}{lll}0 & 2 & 0\end{array}$

$\begin{array}{lll}0 & 1 & 0\end{array}$

$\begin{array}{lll}0 & 0 & 1\end{array}$

$\begin{array}{lll}1 & 0 & 1\end{array}$

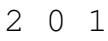

$\begin{array}{lll}2 & 1 & 1\end{array}$

$\begin{array}{lll}1 & 1 & 1\end{array}$

$\begin{array}{lll}1 & 2 & 1\end{array}$

$\begin{array}{lll}0 & 2 & 1\end{array}$

$\begin{array}{lll}0 & 1 & 1\end{array}$

$\begin{array}{lll}0 & 0 & 2\end{array}$

$\begin{array}{lll}1 & 0 & 2\end{array}$

$\begin{array}{lll}1 & 1 & 2\end{array}$

$\begin{array}{lll}0 & 1 & 2\end{array}$

$\begin{array}{lllll}4 & 0 & 7 & 4 & 1\end{array}$

$\begin{array}{lllll}4 & 7 & 6 & 5 & 4\end{array}$

$\begin{array}{lllll}4 & 1 & 4 & 3 & 2\end{array}$

$\begin{array}{lllll}4 & 0 & 1 & 9 & 8\end{array}$

$\begin{array}{llllll}4 & 8 & 9 & 17 & 16\end{array}$

$\begin{array}{lllll}4 & 1 & 2 & 10 & 9\end{array}$

$\begin{array}{lllll}4 & 0 & 8 & 15 & 7\end{array}$

$\begin{array}{lllll}4 & 7 & 15 & 14 & 6\end{array}$

$\begin{array}{lllll}4 & 8 & 16 & 19 & 15\end{array}$

$\begin{array}{lllll}4 & 16 & 17 & 18 & 19\end{array}$

$\begin{array}{lllll}4 & 17 & 9 & 12 & 18\end{array}$

$\begin{array}{lllll}4 & 15 & 19 & 18 & 12\end{array}$

$\begin{array}{lllll}4 & 9 & 10 & 11 & 12\end{array}$

$\begin{array}{lllll}4 & 12 & 13 & 14 & 15\end{array}$

$\begin{array}{lllll}4 & 11 & 10 & 2 & 3\end{array}$

$\begin{array}{lllll}4 & 4 & 12 & 11 & 3\end{array}$

$\begin{array}{lllll}4 & 4 & 5 & 13 & 12\end{array}$

$\begin{array}{lllll}4 & 5 & 6 & 14 & 13\end{array}$

Fig. 11 shows the original PM with 18 quads and the resulting $G^{1}$ PACSIS. 


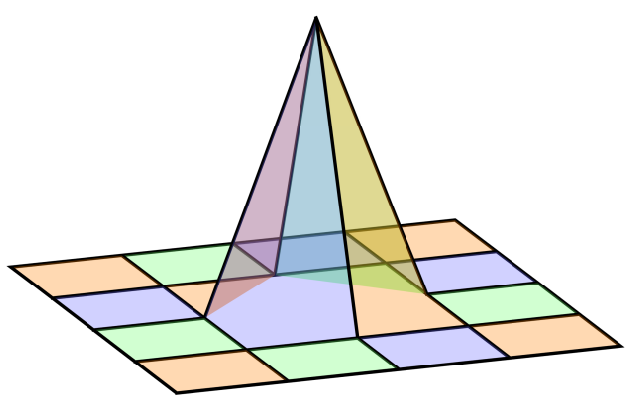

(a)

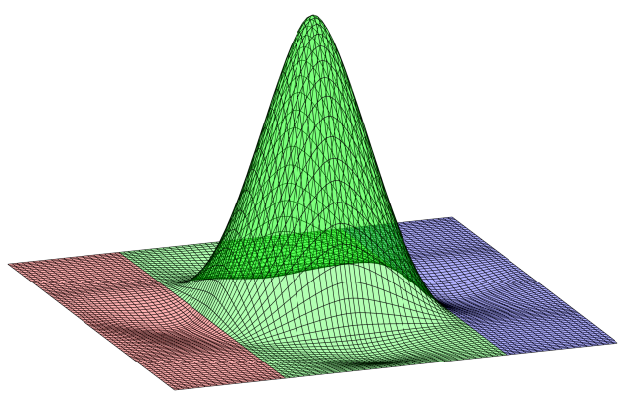

(b)

Fig. 9: (a) A PM with 25 vertices and 16 quads; (b) its resulting PACSIS. Here the global elastic parameter was set as 0.25.

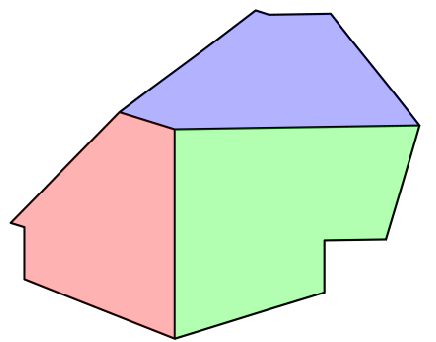

(a)

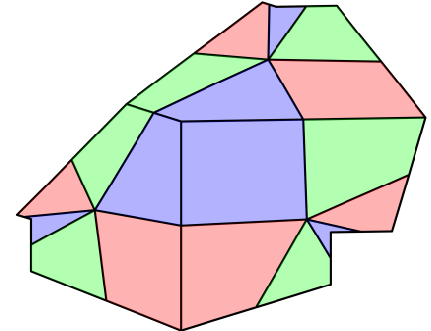

(b)

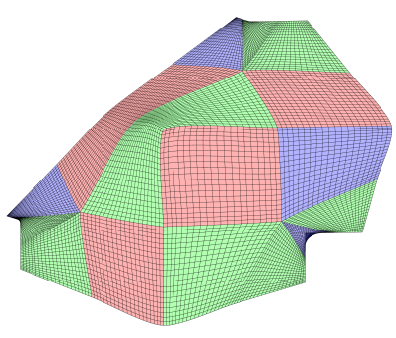

(c)

Fig. 10: (a) A PM with 13 vertices and 3 hexagons, (b) its quadrilateralization, and (c) the resulting PACSIS, Here the global elastic parameter was set as 0.25 .
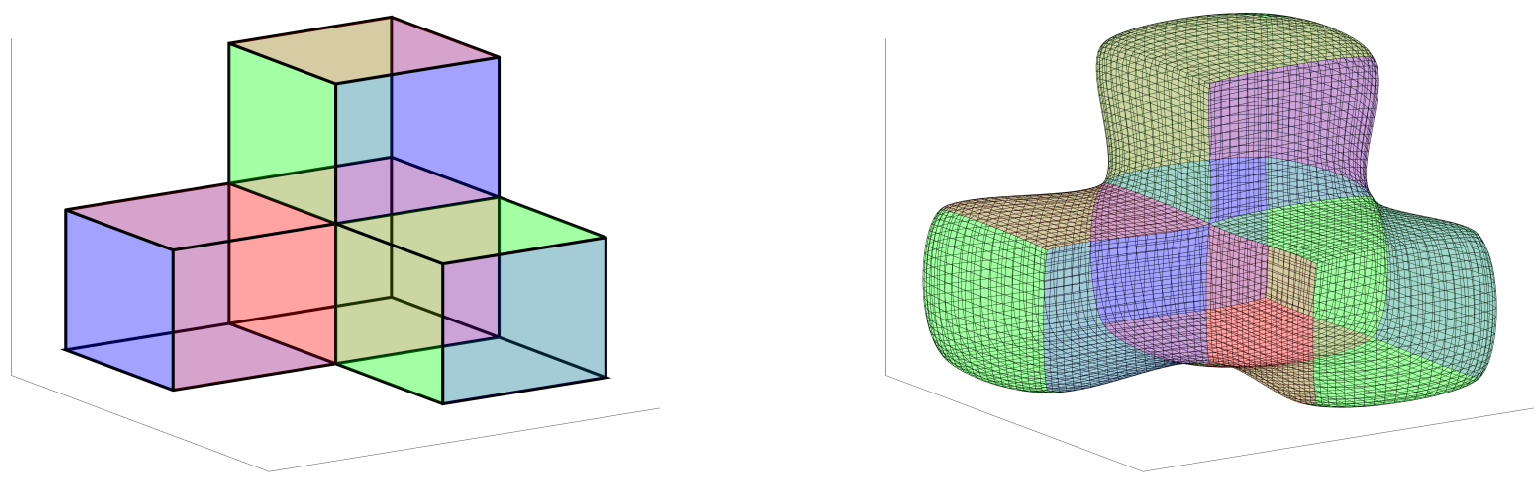

Fig. 11: (a) The PM with 20 vertices and 18 quads and (b) the resulting PACSIS, where the global elastic parameter set as 0.25 . At each corner the surface looks like a cusp but it indeed maintains the $G^{1}$ smoothness. 
Example 4. The PM for a teapot with 1,177 vertices \& 2,256 triangles [15]. The new PLY file contains 6,865 vertices and 6,708 quads after quadrilateralization. The resulting PACSIS is plotted in Fig. 12 (c).

Example 5. The PM for the resolution-2 Stanford bunny with 8,171 vertices \& 16,301 triangles [14]. with the confidence and intensity for each vertex being ignored here. Quadrilateralization preprocessing yields a new PLY file with 48,835 vertices and 48,903 quads. The resulting PACSIS is shown in the last image in Fig. 13

In addition to Example 5, a new PLY file has also been created, with 209,686 vertices and 208,353 quads after quadrilateralization, from the PM of the highest resolution Stanford bunny with 35,947 vertices \& 69,451 triangles [14]. Due to the huge size of the resulting PACSIS by Algorithm 1, Fig. 14 (a) only shows the point cloud from 209,686 vertices of the 208,353 quads. For comparison purposes, Fig. 14 (b) shows the point cloud of the PACSIS from the resolution-4 Stanford bunny with 453 vertices \& 948 triangles. The point cloud has 139,356 points when each PACS is represented by 49 points (on a $7 \times 7$ partitioned curves).

\section{CONCLUSION}

An innovative and efficient algorithm was established for generating a $G^{1}$ surface for any polygonal mesh by using bicubic spline patches. If not all polygons of the given polygonal mesh is quadrilaterals, a quadrilateralization preprocessing is needed. The resulting $G^{1}$ surface interpolates all vertices of the quads; and the $G^{1}$ smoothness was guaranteed by the special creation of new control points for each quad so that all tangent planes at any vertex match. The algorithm was demonstrated by a variety of PLY files representing different polygonal meshes. No subdivision schemes were used on a given polygonal mesh but a $G^{1}$ surface was generated, which was both $G^{1}$ and explicitly given by piecewise polynomials. Future research will be on (1) integrating additional features of a PLY data file to PACSIS such as confidence, intensity, and vertex and/or face normals; (2) establishing algorithms for locally least square PACSIS so that it is close to the original PM; (3) creating point clouds for various $3 \mathrm{~d}$ models from their associated PACSIS; and (4) finding more applications of PACSIS to areas such as signal and image processing, animation, data science, feature representation, mesh rendering, visualization, as well as computer graphics.

\section{REFERENCES}

[1] M. Amersdorfer AND T. Meurer, Equidistant tool path and cartesian trajectory planning for robotic machining of curved freeform surfaces, in IEEE Transactions on Automation Science and Engineering, 2021, Early Access, doi: 10.1109/TASE.2021.3117691.

[2] E. B. Ameur, D. Sbibih, A. Almhdie, AND C. Leger, New spline quasi-interpolant for fitting 3-D data on the sphere: applications to medical imaging, in IEEE Signal Processing Letters, vol. 14, no. 5, pp. 333-336, May 2007, doi: 10.1109/LSP.2006.888261.

[3] C. DE Boor, A Practical Guide to Splines, Revised Ed., Applied Mathematical Sciences \#27, Springer, 2007
[4] G. Chaikin, An algorithm for high speed curve generation, Computer Graphics and Image Processing, vol. 3, 1974, pp. 346-349, https://doi.org/10.1016/0146-664X(74)90028-8.

[5] C.K. ChuI, Multivariate Splines, CBMS-NSF Regional Conference Series \#54, SIAM, Philadelphia, 1988.

[6] D. N. GRUJIĆ, Numerical Hilbert transform algorithm for causal interpolation of functions represented by cubic and exponential splines, in IEEE Access, vol. 9, pp. 136702-136709, 2021, doi: 10.1109/ACCESS.2021.3117978.

[7] M. JANČIČ, V. CvRTILA, AND G. KoseC, Discretized boundary surface reconstruction, in 2021 44th International Convention on Information, Communication and Electronic Technology (MIPRO), 2021, pp. 278283, doi: 10.23919/MIPRO52101.2021.9596965.

[8] S.A.A. KARIM, Rational bi-quartic spline with six parameters for surface interpolation with application in image enlargement, in IEEE Access, vol. 8, pp. 115621-115633, 2020, doi: 10.1109/ACCESS.2020.3002387.

[9] C. A. MicchelLI, Interpolatory subdivision schemes and wavelets, Journal of Approximation Theory, vol. 86, no. 1, pp. 41-71, https://doi.org/10.1006/jath.1996.0054.

[10] H. Ren and K. Fujisawa, G2 B-spline computation for continuous trajectory generation, in 2021 6th Asia-Pacific Conference on Intelligent Robot Systems (ACIRS), 2021, pp. 1-7, doi: 10.1109/ACIRS52449.2021.9519319.

[11] R. T. Rodrigues, N. Tsiogkas, A. Pascoal, and A. P. Aguiar, Online range-based SLAM using B-spline surfaces, in IEEE Robotics and Automation Letters, vol. 6, no. 2, pp. 1958-1965, April 2021, doi: 10.1109/LRA.2021.3060672.

[12] I.J. SCHOENBERG, Contributions to the problem of approximation of equidistant data by analytic functions, in Quart. Appl. Math., vol. 4. no.1, pp. 45-99 and no.2, 112-141, 1946.

[13] O. Shumeiko AND D. KRAVTSOv, Surface approximation using average interpolating splines, in 2019 IEEE 5th International Conference Actual Problems of Unmanned Aerial Vehicles Developments (APUAVD), 2019, pp. 262-264, doi: 10.1109/APUAVD47061.2019.8943930.

[14] THE STANFORD 3D SCANNING REPOSITORY, http://graphics.stanford. edu/data/3Dscanrep/

[15] Teapot, TEAPOT.PLY, https://people.sc.fsu.edu/ jburkardt/data/ply/ply. html \& https://github.com/gnomeby/canvas3D/blob/master/teapot.ply

[16] S. Wirges, K. Rösch, F. Bieder and C. STILler, Fast and robust ground surface estimation from LiDAR measurements using uniform B-Splines, in 2021 IEEE 24th International Conference on Information Fusion (FUSION), 2021, pp. 1-7, doi: 10.1109/APUAVD47061.2019.8943930.

[17] F. Williams, M. TRager, J. Bruna, and D. ZoRin, Neural splines: fitting 3D surfaces with infinitely-wide neural networks, in 2021 IEEE/CVF Conference on Computer Vision and Pattern Recognition (CVPR), 2021, pp. 9944-9953, doi: 10.1109/CVPR46437.2021.00982.

[18] Y. ZhOU, F. MU, AND J. Hu, Adaptive state updating particle filter tracking algorithm based on cubic spline interpolation, in 2021 International Conference on Electronic Information Engineering and Computer Science (EIECS), 2021, pp. 484-488, doi: 10.1109/EIECS53707.2021.9588085. 


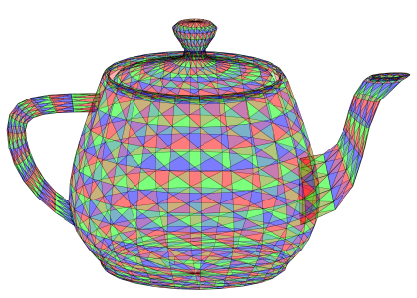

(a)

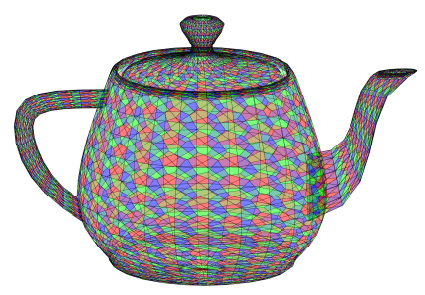

(b)

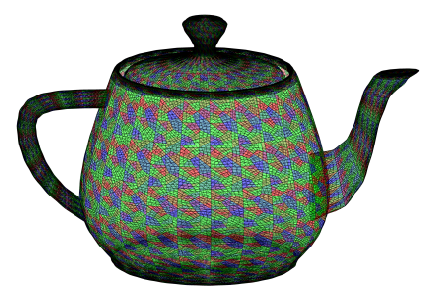

(c)

Fig. 12: (a) The original PM for a teapot with 1,177 vertices \& 2,256 triangles; (b) new PM with 6,865 vertices and 6,708 quads after quadrilateralization; (c) the $G^{1}$ PACSIS of the teapot via Algorithm 1, where each PACS is simply plotted by $4 \times 4$ curves.

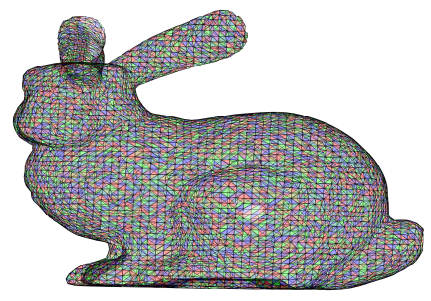

(a)

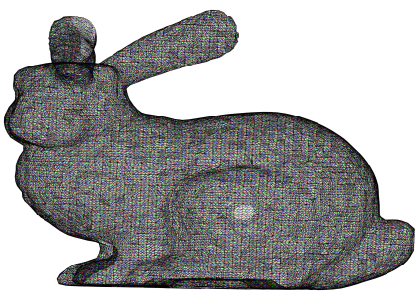

(b)

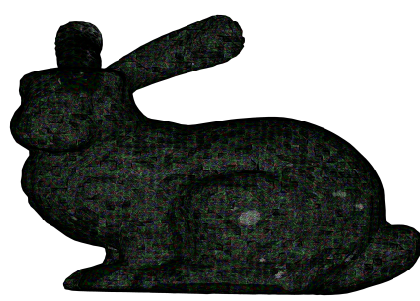

(c)

Fig. 13: (a) The PM for the resolution-2 Stanford bunny with 8,171 vertices \& 16,301 triangles; (b) a new PLY created, with 48,835 vertices \& 48,903 quads after its quadrilateralization preprocessing; (c) the resulting PACSIS by Algorithm 1, with the global elastic parameter being 0.25 and each PACS being plotted by $4 \times 4$ curves. All PACS in the PACSIS in (c) were plotted with 3 alternative colors (RGB). Though three colors were used, it looks black due to the large number of PACS, namely: 48,903 .

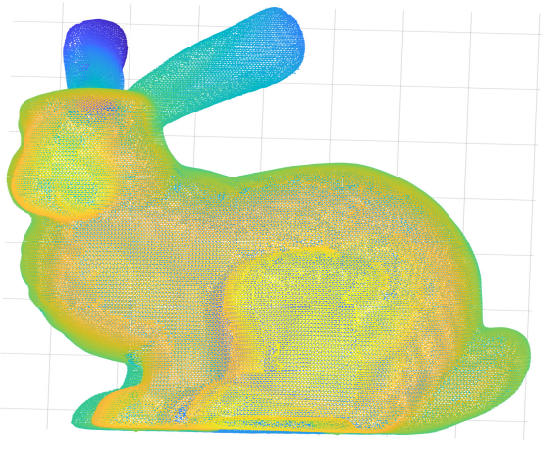

(a)

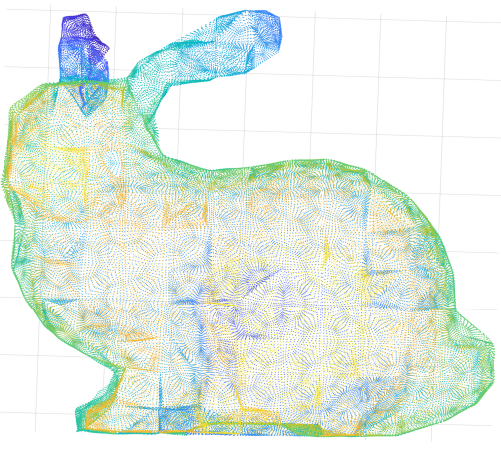

(b)

Fig. 14: (a) A new PLY file is created with 209,686 vertices and 208,353 quads after quadrilateralization for the highest resolution Stanford bunny: 35,947 vertices \& 69,451 triangles [14]. The resulting $G^{1}$ PACSIS by using Algorithm 1, where the global elastic parameter was set as 0.25 , is plotted here by only the point cloud of the PACSIS, or 209,686 points, which is the total number of vertices for all the 208,353 quads; (b) point cloud, or 139,356 points (with slight redundancy), of the PACSIS created from the resolution-4 Stanford bunny with 453 vertices \& 948 triangles. 